\title{
UNIMODALITY AND DOMINANCE FOR SYMMETRIC RANDOM VECTORS \\ BY
}

\author{
MAREK KANTER
}

\begin{abstract}
In this paper a notion of unimodality for symmetric random vectors in $R^{N}$ is developed which is closed under convolution as well as weak convergence. A related notion of stochastic dominance for symmetric random vectors is also studied.
\end{abstract}

1. Introduction. In this paper we explore new notions of unimodality and dominance for random vectors in $R^{N}$ (for exact definitions see \$3). These notions are essentially generalizations of definitions in Sherman [13]. It must be noted, however, that we do not restrict ourselves to random vectors with density as Sherman did; rather, we try to explore our subject in its natural context. In order to accomplish our purpuse we need to delve into the interesting topic of logarithmically concave measures, which we do in $\$ 2$.

In $\$ 3$ we prove our main results. Namely we show that the class of symmetric unimodal probability measures on $R^{N}$ is closed under convolution and weak convergence (where unimodality is defined as in Definition 3.1). We also prove some general results about the notion of symmetric dominance for symmetric unimodal random vectors.

In $\$ 4$ we apply our general results to the study of infinitely divisible random vectors. We give conditions for infinitely divisible random vectors to be symmetric unimodal and as a by-product we see that all symmetric stable random vectors are unimodal. We then make use of semigroup theory in Hille and Phillips [6] to show that if $X(t)$ and $Y(t)$ are homogeneous processes with independent increments with symmetric unimodal Levy-Khinchine measures $M_{X}$ and $M_{Y}$, then $X(t)$ dominates $Y(t)$ for all $t>0$ if and only if $M_{X}$ dominates $M_{Y}$.

In $\$ 5$ we compare our notion of unimodality in $R^{N}$ to three other ways of defining unimodality due to Anderson [1], Olshen and Savage [8], and Ghosh [5].

We end this introduction with a list of basic notation, some definitions and a summary of some required facts about measures and metric spaces.

Received by the editors June 6, 1975.

AMS (MOS) subject classifications (1970). Primary 60D05; Secondary 26A63, 28A75, 52A20.

Key words and phrases. Unimodality, symmetric density, convex set, convolution, logarithmically concave density.

- American Mathematical Society 1977 
Notation. For a vector $a \in R^{N}$ we let $\|a\|$ stand for an arbitrary norm on $R^{N}$, while $\|a\|_{2}$ stands for the usual Euclidean norm $(a \cdot a)^{1 / 2}$. We let $B(r)=\left\{y \mid y \in R^{N},\|y\|_{2} \leqslant r\right\}$.

For $U \subset R^{N}$ we let $I_{U}$ be the indicator function of $U$.

For $U \subset R^{N}, B(U, r)=\left\{y \mid y \in R^{N}, \inf _{x \in U}\|y-x\|_{2} \leqslant r\right\}$.

We let $\operatorname{cl}(U), U^{\circ}, U^{c}$, and $\partial U$ denote the closure of $U$, the interior of $U$, the complement of $U$, and the boundary of $U$ respectively. For $B(1) \subset R^{N}$ we denote $\partial B(1)$ by $S_{N-1}$.

We define $\operatorname{diam}(U)=\sup \left\{\|x-y\|_{2} ; x, y \in U\right\}$.

We let $\operatorname{span}(U)$ be the affine subspace spanned by $U$ and $\operatorname{conv}(U)$ be the least convex set containing $U$.

We let $\operatorname{dim}(U)=\operatorname{dimension}$ of $\operatorname{span}(U)$.

Finally if $U$ is a convex set and $L=\operatorname{span}(U)$, we let $U_{L}^{\circ}$ stand for the interior of $U$ when viewed as a subset of $L$.

Definitions.

DefinITION 1.1. If $L$ is a linear subspace of $R^{N}$ of dimension $k$, we let $\mathrm{Vol}_{L}$ stand for $k$-dimensional Lebesgue measure on $L$, with the normalizing condition that $\operatorname{Vol}_{L}(B(1))=\pi^{k / 2} / \Gamma\left(1+\frac{1}{2} k\right)$.

If $L^{\prime}$ is an affine subspace of $R^{N}$ of the form $L^{\prime}=L+x$ where $L$ is a linear subspace of $R^{N}$ we let $\operatorname{Vol}_{L^{\prime}}\left(A^{\prime}\right)=\operatorname{Vol}_{L}(A)$ where $A^{\prime}=A+x$ and $A$ is any Borel subset of $L$.

Definition 1.2. If $K$ is a compact, convex subset of $R^{N}$ we define the probability measure $\lambda_{K}$ on $R^{N}$ by

$$
\lambda_{K}(A)=\operatorname{Vol}_{L}(A \cap K) / \operatorname{Vol}_{L}(K)
$$

where $A$ is any Borel subset of $R^{N}$ and $L$ is the affine subspace spanned by $K$.

Measures and metric spaces.

Let $\Re$ be a metric space with metric $d$. A measure on $\Re$ is a countably additive $[0, \infty]$ valued set function on the $\sigma$-field of Borel subsets of $\Re$, i.e. those sets generated by the open subsets of $\Re$. A probability measure on $\Re$ is a measure on $\Re$ with $\mu(\Re)=1$.

Definition 1.3. Let $\Re$ be a metric space. Let $\mu_{n}$ be a sequence of bounded measures on $\Re$ and let $\mu$ be a bounded measure on $\Re$. We write $\mu_{n} \rightarrow^{w} \mu$ (and say $\mathfrak{u}_{n}$ converges weakly to $\mu$ ) if

$$
\int f d \mu_{n} \rightarrow f d \mu
$$

for all bounded, continuous, real valued functions on $\Re$. If $X_{n}$ and $X$ are random vectors in $R^{N}$ we say $X_{n}$ converges to $X$ in distribution (and write $\left.X_{n} \rightarrow^{D} X\right)$ if $\mu_{n} \rightarrow^{w} \mu$ where $\mu_{n}$ is the distribution of $X_{n}$ (i.e. $\mu_{n}(A)=P\left[X_{n} \in\right.$ $A$ ] for any Borel subset of $R^{N}$ ) and where $\mu$ is the distribution of $X$. 
It is well known (see Parthasarathy [9]) that if $\mathscr{N}$ is separable then the set of probability measures on $\Re$ may be made into a separable metric space $\left(\Re^{+}, d^{+}\right)$where $d^{+}\left(\mu_{n}, \mu\right) \rightarrow 0$ if and only if $\mu_{n} \rightarrow^{w} \mu$. If $\Re$ is complete then $\mathfrak{R}^{+}$will also be complete. We now mention a useful lemma due to Prokhorov, whose proof can be found in $[9$, p. 47].

LEMMA 1.1. Let $(\mathfrak{T}, d)$ be a complete separable metric space. Let $F=\left\{\mu_{\alpha} \mid \alpha\right.$ $\in A\}$ be a set of probability measures on $\mathfrak{N}$. Then $F$ has compact closure in $\left(\mathfrak{T}^{+}, d^{+}\right)$if and only if for all $\varepsilon>0$ there exists a compact subset $K$ of $\mathfrak{K}$ such that

$$
\mu_{\alpha}(K) \geqslant 1-\varepsilon
$$

for all $\alpha \in A$.

Again let $(\Re, d)$ be a complete separable metric space. Let $\left(\Re^{++}, d^{++}\right)$ denote the set of probability measures on $\Re^{+}$equipped with metric $d^{++}$ which metrizes the notion of weak convergence of probability measures on $\mathfrak{N}^{+}$. Let $F$ be a set of probability measures on $\mathfrak{N}^{+}$. A double application of Lemma 1.1 enables us to conclude that $F$ has compact closure in $\left(\mathfrak{R}^{++}, d^{++}\right)$if and only if for all $\varepsilon_{1}>0, \varepsilon_{2}>0$ there exists a compact subset $K$ of $\mathfrak{T}$ such that for all $\nu \in F$

$$
\nu\left\{\mu \mid \mu \in \Re^{+}, \mu(K) \geqslant 1-\varepsilon_{1}\right\} \geqslant 1-\varepsilon_{2} .
$$

If $\mathscr{N}=R^{N}$ we shall use the notation $\mathfrak{R}^{+}=\Re_{N}^{+}$and $\Re^{++}=\Re_{N}^{++}$. If $\mathfrak{R}=L$, a linear subspace of $R^{N}$, we shall write $\mathfrak{R}^{+}=\mathfrak{R}_{L}^{+}$and $\mathfrak{R}^{++}=$ $\mathfrak{M L}_{L}^{++}$.

Definition 1.4. Let $\mu_{1}, \mu_{2} \in \Re^{+}$. We define the total variation norm between $\mu_{1}$ and $\mu_{2}$ (written $\left\|\mu_{1}-\mu_{2}\right\|_{\text {t.v. }}$ ) to be $\sup _{A}\left|\mu_{1}(A)-\mu_{2}(A)\right|$ where the sup is taken over the family of all Borel subsets $A$ of $\Re$. We write $\mu_{n} \rightarrow^{\text {t.v. }} \mu$ if $\left\|\mu_{n}-\mu\right\|_{\text {t.v. }} \rightarrow 0$. It is well known that $\mu_{n} \rightarrow^{\text {t.v. }} \mu$ implies $\mu_{n} \rightarrow^{w} \mu$. It is also well known that $\left(\mathfrak{R}^{+},\|\|_{\text {t.v. }}\right)$ is a complete metric space, even if $\Re$ is not complete.

Suppose now that $L$ is a linear subspace of $R^{N}$. If $h$ is a Borel measurable real valued function on $L$ we define $\|h\|_{L}=\int_{L} 1 h(x) 1 d \operatorname{Vol}_{L}(x)$. We shall let $\mathcal{L}^{1}\left(\mathrm{Vol}_{L}\right)$ denote the set of all Borel measurable functions $h$ on $L$ with $\|h\|_{L}<\infty$. If $\mu$ is a probability measure on $L$ which is absolutely continuous with respect to $\mathrm{Vol}_{L}$ and has Radon-Nikodym derivative $f$ with respect to $\mathrm{Vol}_{L}$, we shall write $d \mu=f d \mathrm{Vol}_{L}$ or $d \mu / d \mathrm{Vol}_{L}=f$. It is well known that if $\mu, \nu$ are probability measures on $L$ with $d \mu=f d \mathrm{Vol}_{L}$ and $d \nu=g d \mathrm{Vol}_{L}$, then $\|\mu-\nu\|_{\text {t.v. }}=\frac{1}{2}\|f-g\|_{L}$.

DEFINITION 1.5. Let $(\Re, d)$ be a complete separable metric space and let $\mu$ be a measure on $\Re$. We define the support of $\mu($ written $\operatorname{supp}(\mu))$ to be the 
smallest closed subset $D$ of $\mathscr{N}$ such that $\mu\left(D^{c}\right)=0$. Equivalently, $\operatorname{supp}(\mu)$ is the set of all points $x$ in $\Re$ such that $\mu(x+B(a))>0$ for all $a>0$. (See [9, p. 29].)

We end the introduction with a well-known lemma about weak convergence of measures (see [9, p. 40] for the proof).

LEMMA 1.2. Let $(\mathfrak{R}, d)$ be a metric space. Let $\mu_{n}$ be a sequence of bounded measures on $\Re$ with $\mu_{n} \rightarrow^{w} \mu$. Then:

(1) For any closed set $C$ in $\Re$ we have lim $\sup _{n \rightarrow \infty} \mu_{n}(C)<\mu(C)$.

(2) For any Borel subset $A$ of $\Re$ with $\mu(\partial A)=0$ we have $\lim _{n \rightarrow \infty} \mu_{n}(A)=$ $\mu(A)$.

2. Log-concave measures. In this section we present the main facts about an important class of probability measures on $R^{N}$.

Definition 2.1. We say that a probability measure $\mu$ on $R^{N}$ is logarithmically concave if for all open, convex sets $C, D \subset R^{N}$ and $t \in(0,1)$ we have

$$
\mu(t C+(1-t) D)>\left(\mu(C)^{t}\right)\left(\mu(D)^{1-t}\right)
$$

It is easy to deduce that (2.1) is also valid for all closed, convex sets $C, D$, since any closed convex set is the limit of a decreasing sequence of open convex sets. An easy consequence of (2.1) is the inequality

$$
\mu(C-(t x+(1-t) y)) \geqslant\left(\mu(C-x)^{t}\right)\left(\mu(C-y)^{1-t}\right),
$$

valid for all $x, y \in R^{N}$. A direct consequence of (2.2) is the fact that $\operatorname{supp}(\mu)$ is a closed convex set. If we let $L=\operatorname{span}(\operatorname{supp}(\mu))$ it is natural to conjecture that $d \mu=f d \mathrm{Vol}_{L}$ for some logarithmically concave function $f$ on $L$. (A function $f$ is said to be $\log$-concave if $\log f$ has values in $[-\infty,+\infty)$ and is concave.) This conjecture has recently been proved in [2, p. 123]. The converse to this conjecture was demonstrated by Prekopa [10]. We combine these results to get the following key lemma.

LEMMA 2.1. Let $\mu$ be a logarithmically concave measure on $R^{N}$. Then there exists a linear subspace $L$ of $R^{N}$ and a log-concave density function $f$ such that $d \mu=f d \mathrm{Vol}_{L}$. Conversely if $L$ and a log-concave density function $f$ are given, then the measure $\mu$ defined by $d \mu=f d \mathrm{Vol}_{L}$ is logarithmically concave.

COROLlaRY 2.1. For any compact, convex, symmetric set $K$ and any symmetric open, convex set $C$ we have $\lambda_{K}(C-x)<\lambda_{K}(C)$.

Proof. The measure $\lambda_{K}$ has log-concave density $\operatorname{Vol}_{L}(K)^{-1} I_{K}$ where $L=$ $\operatorname{span}(K)$, hence by Lemma $2.1, \lambda_{K}$ is a log-concave measure. Now use (2.2). Q.E.D.

LEMMA 2.2. Suppose $\left(\mu_{n} \mid n \geqslant 1\right)$ is a sequence of log-concave probability measures with $\mu_{n} \rightarrow^{w} \mu$. Then $\mu$ is also a log-concave probability measure. 
Proof. We wish to show that $\mu$ satisfies (2.1). In fact whenever $C, D$ are closed convex sets with nonempty interior and $\mu(\partial C)=\mu(\partial D)=0$, then (2.1) is satisfied since in that case

$$
\mu(C)=\lim _{n \rightarrow \infty} \mu_{n}(C) \text { and } \mu(D) \lim _{n \rightarrow \infty} \mu_{n}(D)
$$

by Lemma 1.2. To demonstrate (2.1) for general open convex sets $C$ and $D$, we note that any open convex set $A$ is the limit of an increasing sequence $A_{n}$, of closed convex sets with nonempty interior which are chosen so that $\mu\left(\partial A_{n}\right)=0$. Q.E.D.

LEMMA 2.3. Let $\mu_{1}$ and $\mu_{2}$ be log-concave probability measures on $R^{N}$. Then $\mu_{1} * \mu_{2}$ is also a log-concave probability measure on $R^{N}$.

Proof. Let $C_{i}=\operatorname{supp}\left(\mu_{i}\right)$ for $i=1,2$. Translating $\mu_{i}$ by vectors $x_{i}$ if necessary, we may suppose $0 \in C_{1} \cap C_{2}$. Let $L_{i}=\operatorname{span}\left(C_{i}\right)$ and let $L_{i}^{\perp}$ be the linear subspaces orthogonal to $L_{i}$ so that $R^{N}=L_{i} \oplus L_{i}^{\perp}$ for $i=1,2$. Let

$$
g_{i, n}\left(y_{i}\right)=\frac{1}{\operatorname{Vol}_{L_{i}}(B(1 / n))} I_{B(1 / n) \cap L_{i}}\left(y_{i}\right) \text { for } y_{i} \in L_{i}^{\perp}
$$

and let

$$
f_{i, n}\left(x_{i}\right)=\frac{1}{\operatorname{Vol}_{L_{1}}(B(1 / n))} \mu_{i}\left(B(1 / n)-x_{i}\right) \text { for } x_{i} \in L_{i} \text {. }
$$

Let $\nu_{i, n}$ be the probability measure on $R^{N}$ with density $f_{i, n} g_{i, n}$ with respect to $\mathrm{Vol}_{L}=\mathrm{Vol}_{L_{1}} \times \mathrm{Vol}_{L_{1}}$. It is proved in [10] as well as [3] that $\nu_{1, n} * \nu_{2, n}$ is a log-concave probability measure.

It is clear that $\nu_{1, n} \rightarrow^{w} \mu_{1}$ and $\nu_{2, n} \rightarrow^{w} \mu_{2}$ as $n \rightarrow \infty$. We conclude that $\nu_{1, n} * \nu_{2, n} \rightarrow{ }^{w} \mu_{1} * \mu_{2}$ and $\mu_{1} * \mu_{2}$ is a log-concave measure, since log-concavity is preserved under weak limits. Q.E.D.

LEMMA 2.4. Let $\mu$ be a log-concave measure on $R^{N}$. Let $L$ be a linear subspace and let $\Pi_{L}$ stand for the orthogonal projection operator onto $L$. Then the measure $\mu \circ \Pi_{L}$ is a log-concave measure on $L$.

Proof. If $C, D$ are open convex subsets of $L$ then $\Pi_{L}^{-1}(C)$ and $\Pi_{L}^{-1}(D)$ are open convex subsets of $R^{N}$. Since $\mu$ is log-concave, $(2.1)$ is satisfied for $\Pi^{-1}(C), \Pi^{-2}(D)$; it follows that $(2.1)$ is satisfied for $\mu \circ \Pi_{L}^{-1}$ and $C, D$. Q.E.D.

Definition 2.2. Let $L$ be a linear subspace of $R^{N}$. We shall denote by $T_{L}$ the set of all log-concave, symmetric nonnegative functions $f$ on $L$ with $\int_{L} f d \mathrm{Vol}_{L}=1$, and we shall denote by $T_{L}^{*}$ the set of all probability measures $\mu$ on $L$ of the form $d \mu=f d \mathrm{Vol}_{L}$ where $f \in T_{L}$.

COROLlaRY 2.3. The set $T_{L}$ is complete separable metric space when equipped with the metric $\|\cdot\|_{L}$ and when functions which agree a.e. $\mathrm{Vol}_{L}$ are identified. 
Proof. Suppose $\left\|f_{n}-f_{m}\right\|_{L} \rightarrow 0$ as $n, m \rightarrow \infty$, where the sequence $f_{n} \in T_{L}$. Then $\left\|\mu_{n}-\mu_{m}\right\|_{\text {t.v. }} \rightarrow 0$, where $\mu_{m}$ is a sequence of probability measures on $L$ defined by $d \mu_{n}=f_{n} d \mathrm{Vol}_{L}$. Now this implies the existence of some probability measure $\mu$ on $L$ such that $\left\|\mu_{n}-\mu\right\|_{\text {t.v. }} \rightarrow 0$ as $n \rightarrow \infty$. In particular, $\mu_{n} \rightarrow{ }^{w} \mu$, hence by Lemma 2.2, $\mu$ is a log-concave measure on $R^{N}$. Now $\mu$ has density with respect to $\mathrm{Vol}_{L}$ since $\left\|\mu_{n}-\mu\right\|_{\text {t.v. }} \rightarrow 0$; also $\mu$ is clearly symmetric. We can now use Lemma 2.1 to conclude that $d \mu / d \mathrm{Vol}_{L}=f$ where $f \in T_{L}$.

We have just proved completeness of $T_{L}^{*}$. The proof of separability is standard. Q.E.D.

3. Symmetric unimodal random vectors. In this section we shall introduce our notion of unimodality for symmetric random vectors in $R^{N}$, and we shall prove a few fundamental results about this notion.

We shall of ten be dealing with sets $\mathcal{S}$ which consist of a subset of the collection of all probability measures on some metric space $\mathfrak{T}$ and we shall need to have a uniform convention for the concept of Borel subsets of $\delta$. Our convention shall be to endow $\delta$ with the Borel structure consisting of the least $\sigma$-field containing all those subsets $U$ of $\delta$ which are open in the topology of weak convergence of probability measures.

Definition 3.1. Let $L$ be a linear subspace of $R^{N}$. We shall let $W_{L}$ stand for the set of all probability measures on $L$ of the form $\lambda_{K}$ where $K$ is a compact symmetric subset of $L$. We give $W_{L}$ the Borel structure mentioned above.

We let $U_{L}$ stand for all the measures $\mu$ on $R^{N}$ which are of the form

$$
\mu=\int_{W_{L}} \lambda_{K} d \nu\left(\lambda_{K}\right)
$$

where $\nu$ is a measure on $W_{L}$. We call $\nu$ the mixing measure for $\mu$. (We shall later show that $W_{L}$ is a Borel measurable subset of $\Re_{L}^{+}$.) We note that $\mu$ in (3.1) is a probability measure if and only if $\nu$ is a probability measure.

We shall use the notation $W_{R^{N}}=W_{N}$ and $U_{R^{N}}=U_{N}$. We shall call a random vector in $R^{N}$, symmetric unimodal if its distribution is in $U_{N}$. We note that if $\mu$ is a symmetric probability measure on $R$ which is unimodal in the standard 1-dimensional sense, then by Feller [4, p. 158] we have $\mu \in U_{1}$.

LEMMA 3.1. Let $L$ be a linear subspace of $R^{N}$ and let $\mu$ be a log-concave, symmetric, probability measure on $L$ with $d \mu / d \mathrm{Vol}_{L}=f$ where $f \in T_{L}$. Then $\mu \in U_{L}$.

Proof. Let $D_{s}=\{y \mid f(y) \geqslant s\}$ for $s>0$. We have $f(x)=\int_{0}^{\infty} I_{D_{s}}(x) d s$; hence it follows that for any Borel subset $A$ of $L$, 


$$
\int_{A} f(x) d \operatorname{Vol}_{L}(x)=\int_{0}^{\infty} \operatorname{Vol}_{L}\left(A \cap D_{s}\right) d s .
$$

Now $D_{s}$ is a bounded convex subset of $L$ for $s>0$ and it is clear that $\operatorname{Vol}_{L}\left(D_{s}\right)=\operatorname{Vol}_{L}\left(K_{s}\right)$ for all $s$, where $K_{s}=\operatorname{cl}\left(D_{s}\right)$. It follows that

$$
\operatorname{Vol}_{L}\left(A \cap D_{s}\right)=\operatorname{Vol}_{L}\left(A \cap K_{s}\right)
$$

for all $s$ and we can rewrite (3.2) as

$$
\int_{A} f(x) d \operatorname{Vol}_{L}(x)=\int_{0}^{\infty} \operatorname{Vol}_{L}\left(A \cap K_{s}\right) d s .
$$

Now let $\Phi$ be the measurable map from $(0, \infty)$ into $W_{L}$ defined by $\Phi(s)=\lambda_{K_{s}}$ and let $\nu^{\prime}=\mathcal{L} \circ \Phi^{-1}$ where $\mathcal{L}$ is Lebesgue measure on $(0, \infty)$. We rewrite (3.3) as

$$
\int_{A} f(x) d \operatorname{Vol}_{L}(x)=\int_{W_{L}} \lambda_{K}(A) \operatorname{Vol}_{L}(K) d \nu^{\prime}\left(\lambda_{K}\right)
$$

Finally we let the measure $\nu$ on $W_{L}$ be defined by the equation

$$
\nu(B)=\int_{B} \operatorname{Vol}_{L}(K) d \nu^{\prime}\left(\lambda_{K}\right)
$$

where $B$ is any Borel subset of $W_{L}$. It is clear that we can rewrite (3.4) as

$$
\int_{A} f(x) d \operatorname{Vol}_{L}(x)=\int_{W_{L}} \lambda_{K}(A) d v\left(\lambda_{K}\right)
$$

hence we conclude that $\mu \in U_{L}$ with mixing measure $\nu$. Q.E.D.

Notation. Let $\mu$. and $\nu$ be as in Lemma 3.1. We shall denote $\nu$ by $J(\mu)$. We claim that $J$ is a well-defined map from $T_{L}^{*}$ into $W_{L}^{+}$. In fact suppose

$$
d \mu=f_{1} d \mathrm{Vol}_{L}=f_{2} d \mathrm{Vol}_{L}
$$

where $f_{1}=f_{2}$ a.e. $\left(\operatorname{Vol}_{L}\right)$ and $f_{1}=f_{2} \in T_{L}$. It is then obvious that the two compact convex sets $\operatorname{cl}\left\{y \mid f_{1}(y)>s\right\}$ and $\operatorname{cl}\left\{y \mid f_{2}(y) \geqslant s\right\}$ are equal for all $s>0$.

We shall study the measurability properties of the map $J$ in the following lemma.

LeMMA 3.2. The map $J: T_{L}^{*} \rightarrow W_{L}^{+}$is measurable.

Proof. We must show that for any Borel set $C$ of $W_{L}^{+},\{\mu \mid J(\mu) \in C\}$ is a Borel subset of $T_{L}^{*}$. It suffices to consider sets $C$ of the form

$$
C=\left\{\nu \mid \int_{W_{L}} k\left(\lambda_{k}\right) d \nu\left(\lambda_{K}\right)>r\right\}
$$

where $h$ is a bounded continuous function on $W_{L}$ and $r \in R$. We can assume $h\left(\lambda_{K}\right)=\int_{L} g d \lambda_{K}$ where $g$ is a bounded continuous function on $L$. 
We know that any $\mu \in T_{L}^{*}$ is of the form $d \mu=f_{\mu} d$ Vol $_{L}$ where $f_{\mu}$ is a logarithmically concave density function. Let us set $K_{\mu, s}=\left\{y \mid f_{\mu}(y)>s\right\}$. We now compute

$$
\int_{W_{L}} h\left(\lambda_{K}\right) d \nu\left(\lambda_{K}\right)=\int_{W_{L}}\left(\int_{L} g d \lambda_{K}\right) \operatorname{Vol}_{L}(K) d \nu^{\prime}(K),
$$

whence

$$
\int_{W_{L}} h\left(\lambda_{K}\right) d \nu\left(\lambda_{K}\right)=\int_{W_{L}} \int_{K} g d \operatorname{Vol}_{L} d \nu^{\prime}(K) .
$$

Remember now that $\nu^{\prime}=\mathfrak{L} \circ \Phi_{\mu}^{-1}$ where $\Phi_{\mu}(s)=\lambda_{K_{\mu,}}$; then the last expression becomes $\int_{0}^{\infty}\left(\int_{K_{\mu, s}} g d \mathrm{Vol}_{L}\right) d s$.

It follows that

$$
\{\mu \mid J(\mu) \in C\}=\left\{\mu \mid \int_{0}^{\infty}\left(\int_{K_{\mu, S}} g d \mathrm{Vol}_{L}\right) d s>r\right\} .
$$

Using (3.2) we get

$$
\{\mu \mid J(\mu) \in C\}=\left\{\mu \mid \int_{L} g(x) f_{\mu}(x) d \operatorname{Vol}_{L}(x)>r\right\} .
$$

Hence,

$$
\{\mu \mid J(\mu) \in C\}=\left\{\mu \mid \int_{L} g d \mu>r\right\} .
$$

The last set is clearly a Borel subset of $T_{L}^{*}$. Q.E.D.

COROLlaRy 3.1. $W_{L}$ is a Borel subset of $\pi_{L}^{+}$.

Proof. We have $W_{L} \subset T_{L}^{*} \subset \Re_{L}^{+}$.

Using Corollary 2.3 we know that $T_{L}^{*}$ is a complete metric space under the total variation metric; also the imbedding $T_{L}^{*} \subset \Re_{L}^{+}$is continuous where $T_{L}^{*}$ is given the topology determined by the total variation metric while $\mathfrak{T}_{L}^{+}$is given the topology determined by weak convergence of probability measures. We conclude by a theorem of Kuratowski [7, p. 21] that $T_{L}^{*}$ is a Borel subset of $\pi_{L}^{+}$.

Consider now the set $H \subset W_{L}^{+}$defined by $H=\{\mu \mid \mu$ is an atomic probability measure on $\left.W_{L}\right\}$. Now $W_{L}$ is separable under the topology of weak convergence; hence there exists a sequence $A_{n}$ of Borel subsets of $W_{L}$ such that $H=\left\{\mu \mid \mu \in \Re_{L}^{+}, \mu\left(A_{n}\right)=0\right.$ or 1 for all $\left.n\right\}$. We conclude $H$ is a Borel subset of $W_{L}^{+}$. Noting $W_{L}=J^{-1}(H)$, by Lemma 3.2 we conclude that $W_{L}$ is a Borel subset of $T_{L}^{*}$. Since $T_{L}^{*}$ is a Borel subset of $\mathfrak{T}_{L}^{+}$, we are done. Q.E.D. 
The next theorem is one of the fundamental results in this paper, namely that symmetric unimodal probability measures are closed under weak convergence. It is easy to prove in 1 dimension; its difficulty in $N$ dimensions is essentially due to the fact that $\bar{W}_{N}$, the closure of $W_{N}$ in $\Re_{N}$ in the topology of weak convergence, contains $W_{N}$ properly. The following example will clarify things.

EXAMPLE 3.1. In $R^{2}$ let

$$
K_{n}=\operatorname{conv}(\{(1,0),(-1,0),(0,1 / n),(0,-1 / n)\}) .
$$

A simple computation shows that $\lambda_{K_{n}} \rightarrow^{w} \mu$ where $\mu$ is the probability measure on $L=\{(x, y) \mid y=0\}$ whose density is $|1-x|$ on $K=\{(x, y) \mid x \in$ $[-1,1], y=0\}$ with respect to $\mathrm{Vol}_{L}$.

THEOREM 3.1. The class of symmetric unimodal probability measures on $R^{N}$ is closed under weak convergence.

PRoOF. Suppose $\mu_{n}$ is a sequence of probability measures in $U_{N}$ with $\mu_{n} \rightarrow^{w} \mu$ where $\mu$ is a probability measure on $R^{N}$. By Lemma 1.1 we know that for any $\theta, \delta>0$ there exists $n$ so large that $\mu_{n}(B(r))>1-(\theta / \delta)$ for all $n$ as well as $\mu(B(r))>1-\theta / \delta$. Now we can write

$$
\mu_{n}=\int_{W_{N}} \lambda_{K} d \nu_{n}\left(\lambda_{K}\right)
$$

for some probability measure $\nu_{n}$ on $W_{N}$. We conclude that $\nu_{n}\left\{\lambda_{K}\left|\lambda_{K}(B(r))^{c}\right\rangle\right.$ $\left.\delta^{-1}\right\}<\theta$. Let $\bar{W}_{N}$ stand for the closure of $W_{N}$ in $\mathfrak{N}_{N}$. Applying (1.5) we conclude immediately that the set $\left\{\nu_{n} \mid n \geqslant 1\right\}$ has compact closure in $\left(\bar{W}_{N}\right)^{+}$, so by passing to a subsequence if necessary, we know there exists $\bar{\nu} \in\left(\bar{W}_{N}\right)^{+}$ such that $\nu_{\mathbf{k}} \stackrel{\sim}{\rightarrow} \bar{\nu}$. This means that

$$
\int_{\bar{W}_{n}} f d \nu_{n} \rightarrow \int_{\bar{W}_{N}} f d \bar{\nu}
$$

for all bounded continuous real valued functions $f$ on $\bar{W}_{N}$. Now $\bar{W}_{N}$ is clearly a measurable subset of $T_{N}^{*}$ (since it is a complete separable metric space and the theorem of Kuratowski [9, p. 21] applies), so we conclude by Lemma 3.2 that the restriction $J: \bar{W}_{N} \rightarrow W_{N}^{+}$is a measurable map. It follows that any probability measure $Q$ on $\bar{W}_{N}$ induces a probability measure $Q \circ J^{-1}$ on $W_{N}^{+}$. In (3.13) let us take $f(\alpha)=\int_{R^{N}} g(x) d \alpha(x)$ where $\alpha \in \bar{W}_{N}$ and $g$ is a bounded continuous real function on $R^{N}$. We can rewrite (3.13) as

$$
\begin{aligned}
\lim _{n \rightarrow \infty} \int_{W_{N}^{+}}\left[\int_{W_{N}}\left\{\int_{R^{N}} g d \lambda_{K}\right\} d \beta\left(\lambda_{K}\right)\right] d \nu_{n} \circ J^{-1}(\beta) \\
=\int_{W_{N}^{+}}\left[\int_{W_{N}}\left\{\int_{R^{N}} g d \lambda_{K}\right\} d \beta\left(\lambda_{K}\right)\right] d \bar{\nu} \circ J^{-1}(\beta) .
\end{aligned}
$$


It follows that for all such $g$,

$$
\int_{R^{N}} g(x) d \mu(x)=\int_{W_{N}^{+}}\left[\int_{W_{N}}\left\{\int_{R^{N}} g d \lambda_{K}\right\} d \beta\left(\lambda_{K}\right)\right] d\left(\bar{\nu} \circ J^{-1}\right)(\beta) .
$$

We now define $\nu \in W_{N}^{+}$by the equation

$$
\int_{W_{N}} h\left(\lambda_{K}\right) d \nu\left(\lambda_{K}\right)=\int_{W_{N}^{+}}\left[\int_{W_{N}}\left\{\int_{R^{N}} g d \lambda_{K}\right\} d \beta\left(\lambda_{K}\right)\right] d\left(\bar{\nu} \circ J^{-1}\right)(\beta)
$$

valid for all bounded continuous real valued functions on $W_{N}$ of the form $h\left(\lambda_{K}\right)=\int_{R^{N}} g d \lambda_{K}$. Putting (3.13)-(3.16) together we conclude that $\mu=$ $\int_{W_{N}} \lambda_{K} d \nu\left(\lambda_{K}\right)$. Q.E.D.

We now prove that symmetric unimodal probability measures on $R^{N}$ are closed under convolution. This closure property is a strong justification for our belief that we are working with the proper notion of unimodality for symmetric random vectors in $R^{N}$. It is well known to be valid in one dimension (note however that the convolution of nonsymmetric unimodal probability measures is not necessarily unimodal as shown in [4, p. 168]).

Theorem 3.2. Let $\mu_{1}, \mu_{2} \in U_{N}$. Then $\mu_{1} * \mu_{2} \in U_{N}$.

Proof. We can write $\mu_{i}=\int_{W_{N}} \lambda_{K} d \nu_{\mathrm{i}}\left(\lambda_{K}\right)$ where $\nu_{i}$ are probability measures on $W_{N}$ for $i=1,2$. We conclude that

$$
\mu_{1} * \mu_{2}=\int \lambda_{K_{1}} * \lambda_{K_{2}} d \nu_{1}\left(\lambda_{K_{1}}\right) \times \nu_{2}\left(\lambda_{K_{2}}\right)
$$

Now $\lambda_{K_{1}} * \lambda_{K_{2}}$ is a symmetric log-concave probability measure on $R^{N}$ by Lemma 2.6, hence $\lambda_{K_{1}} * \lambda_{K_{2}} \in U_{N}$, so we can write

$$
\lambda_{K_{1}} * \lambda_{K_{2}}=\int_{W_{N}} \lambda_{K} d \nu_{K_{1}, K_{2}}\left(\lambda_{K}\right)
$$

for some probability measure $\nu_{K_{1}, K_{2}}$ on $W_{N}$. Finally we can write $\mu_{1} * \mu_{2}=$ $\int_{W_{N}} \lambda_{K} d \nu\left(\lambda_{K}\right)$ where $\nu$ is the probability measure on $W_{N}$ defined by

$$
\nu(B)=\int_{W_{n} \times W_{n}} \nu_{K_{1}, K_{2}}(B) d \nu_{1}\left(\lambda_{K_{1}}\right) \times d \nu_{2}\left(\lambda_{K_{2}}\right),
$$

for all Borel subsets $B$ of $W_{N}$. Q.E.D.

The following closure property is an easy corollary to Lemma 2.7. We state it for completeness, but omit the proof.

THEOREM. 3.3. Let $\mu \in U_{N}$. Let $L$ be a linear subspace of $R^{N}$ and let $\Pi_{L}$ denote the orthogonal projection operator onto $L$. Then the measure $\mu \circ \Pi_{L}^{-1}$ is in $U_{L}$.

An important corollary of the last theorem is that all marginals of a symmetric unimodal measure on $R^{N}$ are also symmetric unimodal measures. 
We now turn to the notion of dominance.

Definition 3.3. Let $\mu_{1}$ and $\mu_{2}$ be measures on $R^{N}$. We say that $\mu_{1}$ symmetrically dominates $\mu_{2}$ (and write $\mu_{1}>\mu_{2}$ ) if we have

$$
\mu_{1}\left(K^{c}\right) \geqslant \mu_{2}\left(K^{c}\right)
$$

for all compact, convex, symmetric subsets of $R^{N}$. If $X_{1}$ and $X_{2}$ are random vectors in $R^{N}$ with distribution $\mu_{1}$ and $\mu_{2}$, respectively, we write $X_{1}>X_{2}$ if $\mu_{1} \succ \mu_{2}$.

Let us denote by $\mathscr{F}_{N}$ the class of continuous, symmetric log-concave functions $f$ on $R^{N}$ with values in $[0, \infty)$ such that $f(0)=1$ and $\lim _{\|x\|_{2} \rightarrow \infty} f(x)$ $=0$.

LEMMA 3.3. Let $\mu_{1}$ and $\mu_{2}$ be measures on $R^{N}$ with $\mu_{i}\left(D^{c}\right)<\infty$, for any Borel measurable set $D$ containing 0 in its interior. Then the condition that $\mu_{1}>\mu_{2}$ is equivalent to the condition that

$$
\int_{R^{N}}(f(0)-f(x)) d \mu_{1}(x) \geqslant \int_{R^{N}}(f(0)-f(x)) d \mu_{2}(x)
$$

for all $f \in \mathscr{F}_{N}$.

Proof. Assume first that $\mu_{1} \succ \mu_{2}$. For $f \in \mathscr{F}_{N}$ let $f_{n}=$ $\sum_{1}^{n}(k / n) I_{\{x \mid f(x)>k / n\}}$. Now $f_{n}$ decreases to $f$ and (3.20) holds for $f_{n}$. We conclude that (3.20) holds for $f$.

Arguing backwards we now assume (3.21) for all $f \in \mathscr{F}_{N}$. Let $K$ be a compact, symmetric convex subset of $R^{N}$ containing 0 in its interior. Let $D_{s}=\partial(s K)$. The sets $D_{s}$ are all disjoint, hence except for $s$ in a countable set $N$ we have $\left(\mu_{1}+\mu_{2}\right)\left(D_{s}\right)=0$.

Define for $\theta>0$,

$$
f_{\theta, s}(x)=\frac{1}{\operatorname{Vol}_{L}(B(\theta))} \operatorname{Vol}_{L}((B(\theta)-x) \cap s K) .
$$

It is clear that $f_{\theta, s} \in \mathscr{F}_{N}$ and that $f_{\theta, s}(\mathrm{x})=1$ if $x \in(s K)^{\circ}$, for $\theta$ sufficiently small. Also for $x \notin s K$, it is clear that $f_{\theta, s}(x)=0$ for $\theta$ sufficiently small. Now we let $\theta \rightarrow 0$ in (3.22) (and use the Lebesgue dominated convergence theorem), in the following inequality,

$$
\int_{R^{N}}\left(f_{\theta, s}(0)-f_{\theta, s}(x)\right) d \mu_{1}(x) \geqslant \int_{R^{N}}\left(f_{\theta, s}(0)-f_{\theta, s}(x)\right) d \mu_{2}(x)
$$

to get that $\mu_{1}\left((s K)^{c}\right) \geqslant \mu_{2}\left((s K)^{c}\right)$ for $s \notin N$. Letting $s \rightarrow 1, s \notin N$, we then get $\mu_{1}\left(K^{c}\right) \geqslant \mu_{2}\left(K^{c}\right)$.

If $K$ has an empty interior, we apply the previous argument to get $\mu_{1}\left(B(K, \delta)^{c}\right) \geqslant \mu_{2}\left(B(K, \delta)^{c}\right)$ for any $\delta>0$. Letting $\delta \rightarrow 0$ we conclude $\mu_{1}\left(K^{c}\right) \geqslant \mu_{2}\left(K^{c}\right)$. Q.E.D. 
LEMMA 3.4. Suppose $\mu \in U_{N}$ and that $\mu_{1}$ and $\mu_{2}$ are two measures on $R^{N^{\prime}}$ with $\mu_{1} \succ \mu_{2}$. Then $\mu \times \mu_{1} \succ \mu \times \mu_{2}$.

Proof. It is sufficient to prove the lemma for $\mu=\lambda_{K} \in W_{N}$, since the general result will follow by mixing. Let $h$ be in $\mathscr{F}_{N+N^{\prime}}$. We compute

$$
\begin{aligned}
\int_{R^{N} \times R^{N^{\prime}}} h\left(0_{N}, 0_{N^{\prime}}\right)-h(x, y) d \lambda_{K}(x) d \mu_{i}(y) \\
=\int_{R^{N^{\prime}}} f\left(0_{N^{\prime}}\right)-f(y) d \mu_{i}(y)
\end{aligned}
$$

where $0_{N}$ and $0_{N^{\prime}}$ stand for the zero vectors in $R^{N}$ and $R^{N^{\prime}}$, respectively, while

$$
f(y)=\int_{R^{N}} h(x, y) d \lambda_{K}(x) .
$$

Now $f$ is a log-concave function on $R^{N^{\prime}}$ by Prekopa [10]; it is obviously continuous symmetric and satisfies $\lim _{\|x\|_{2} \rightarrow \infty} f(x)=0$. Using (3.21) we get that

$$
\begin{aligned}
\int_{R^{N} \times R^{N^{\prime}}}(h(0)-h(z)) & \left(d \lambda_{K} \times d \mu_{1}\right)(z) \\
& \geqslant \int_{R^{N} \times R^{N^{\prime}}}(h(0)-h(z))\left(d \lambda_{K} \times d \mu_{2}\right)(z) . \quad \text { Q.E.D. }
\end{aligned}
$$

CoRollary 3.2. Suppose $\left(\mu_{i} \times \mu_{i}^{\prime}\right)$ for $i=1,2$, are measures on $R^{N} \times R^{N^{\prime}}$. Suppose $\mu_{1} \succ \mu_{2}, \mu_{1}^{\prime}>\mu_{2}^{\prime}$ and that $\mu_{1} \in U_{N}, \mu_{2}^{\prime} \in U_{N^{\prime}}$. Then $\left(\mu_{1} \times \mu_{1}^{\prime}\right)>$ $\left(\mu_{2} \times \mu_{2}^{\prime}\right)$.

Proof. By Lemma 3.4 we know that $\left(\mu_{1} \times \mu_{1}^{\prime}\right)>\left(\mu_{1} \times \mu_{2}^{\prime}\right)$ and that $\left(\mu_{1} \times \mu_{2}^{\prime}\right) \succ\left(\mu_{2} \times \mu_{2}^{\prime}\right)$. We conclude that $\left(\mu_{1} \times \mu_{1}^{\prime}\right) \succ\left(\mu_{2} \times \mu_{2}^{\prime}\right)$. Q.E.D.

The following corollary is a generalization of Lemma 3 in Sherman [13].

Corollary 3.3. Suppose $\left(\mu_{i} \times \mu_{i}^{\prime}\right)$ for $i=1,2$, satisfy the hypothesis of Corollary 3.2, where $N \equiv N^{\prime}$. Then $\mu_{1} * \mu_{1}^{\prime}>\mu_{2} * \mu_{2}^{\prime}$.

Proof. Let $Q: R^{N} \times R^{N} \rightarrow R^{N}$ stand for the addition operator $Q(x, y)=$ $x+y$. If $f \in \mathscr{F}_{N}$ we note that $f \circ Q$ is in $\mathscr{F}_{N+N}$. The rest of the proof is straightforward using Corollary 3.2. Q.E.D.

4. Applications to infinitely divisible symmetric random vectors. In this section we study the notions of symmetric unimodality and symmetric dominance for infinitely divisible random vectors.

Definition 4.1. A symmetric random vector $X$ in $R^{N}$ is infinitely divisible if there exists a time homogeneous stochastic process $(X(t) \mid t>0)$ with symmetric independent increments such that $X(0)=0$ a.s. and $X(1)=X$ a.s. 
It is well known that we can write the characteristic function $\phi_{X}(v)=$ $E\left(e^{i(v \cdot X)}\right)$ of the random vector $X$ in the form (due to Levy and Khinchine)

$$
\log \left(\phi_{X}(v)\right)=\int_{R^{N}}(\cos (v \cdot y)-1) d M_{X}(y)-q_{X}(v)
$$

where $M_{X}$ is a symmetric measure on $R^{N}$ with $M_{X}(\{0\})=0, M_{X}\left(B(a)^{c}\right)<$ $\infty$ and $\int_{B(a)}\left(\|y\|_{2}\right)^{2} d M_{X}(y)<\infty$ for all $a>0$; while $q_{X}(y)=\left(B_{X} y \cdot B_{X} y\right)$ for some symmetric matrix $B_{X}$. (Note that the conditions on $M_{X}$ ensure that $\int\|y-x\|^{2} /\left(1+\|y-x\|^{2}\right) d M_{X}(y)<\infty$ for all $x \in R^{N}$.) We shall assume $q_{X} \equiv 0$, unless otherwise stated (no Gaussian part). The reader is referred to [9] for a derivation of (4.1).

Let $C_{\infty}\left(R^{N}\right)$ denote the set of all bounded infinitely differentiable real valued functions on $R^{N}$. Let us define the operator $T^{t}: C_{\infty}\left(R^{N}\right) \rightarrow C_{\infty}\left(R^{N}\right)$ by

$$
T^{t} f(x)=\int_{R^{N}} f(x-y) d \mu^{t}(y)
$$

where $\mu^{t}$ is the distribution of $X(t)$. The family $\left(T^{t} \mid t>0\right)$ is a semigroup of operators and has an infinitesimal generator $A$ defined by

$$
A f=\lim _{t \rightarrow 0} \frac{1}{t}\left(T^{t}-I\right) f
$$

for any $f$ for which this limit exists in the uniform topology on $C_{\infty}\left(R^{N}\right)$. The class of such functions $f$ is a dense linear subspace $W$ of $C_{\infty}\left(R^{N}\right)$ (see [6]). For $y \in R^{N}$, let

It can also be shown that

$$
\tau(y)=\left\{\begin{array}{ll}
y & \text { if }\|y\|_{2}<1 \\
y /\|y\|_{2} & \text { if }\|y\|_{2}>1
\end{array}\right\}
$$

$$
A f(x)=\int_{R^{N}}(f(x)-f(x-y))-\left(f^{\prime}(x) \cdot \tau(y)\right) d M_{X}(y)
$$

for any $f \in W$. (See $[4$, p. 301].) If we set $x=0$ in (4.4) we get that

$$
\begin{aligned}
\lim _{t \rightarrow 0} \frac{1}{t} & \int_{R^{N}}(f(0)-f(-y)) d \mu^{t}(y) \\
& =\int_{R^{N}}(f(0)-f(-y))-\left(f^{\prime}(0) \cdot \tau(y)\right) d M_{X}(y) .
\end{aligned}
$$

So we conclude

$$
\lim _{t \rightarrow 0} \frac{1}{t} \int_{R^{N}}(f(0)-f(-y)) d \mu^{t}(y)=\int_{R^{N}}(f(0)-f(-y)) d M_{X}(y)
$$

for any $f \in W$, where we have used the fact that $M_{X}$ is a symmetric measure to get the last equality. For the next lemma we need the notation $\nu^{s}=$ $M_{X} * \mu^{s}$. 
LEMMA 4.1. If $f$ is a bounded measurable function which is constant in a neighbourhood of 0 , then

(4.7) $\int_{R^{N}}(f(0)-f(-x)) d \mu^{t}(x)=\int_{0}^{t}\left\{\int_{R^{N}}(f(0)-f(-y)) d \nu^{s}(y)\right\} d s$.

Proof. We first note that for all $a>0$,

$$
\nu^{s}\left(B(a)^{c}\right) \leqslant \mu^{s}\left(B(a / 2)^{c}\right)+M_{X}\left(B(a / 2)^{c}\right)<\infty
$$

and

$$
\int_{B(a)}\left(\|y\|_{2}\right)^{2} d \nu^{s}(y) \leqslant \int_{B(a)}\left(\|y\|_{2}\right)^{2} d \mu^{s}(y)+\int_{B(a)}\left(\|y\|_{2}\right)^{2} d M_{X}(y)<\infty .
$$

Now for any semigroup of operators $\left(T_{t} \mid t>0\right)$ with infinitesimal generator $A$, the formula

$$
\left(T_{t}-I\right)=\int_{0}^{t} A T_{s} d s
$$

is well known (see [6, p. 308]). When we interpret (4.8) in our context we conclude that for any $f \in C_{\infty}\left(R^{N}\right)$ we have

$$
\begin{aligned}
\int_{R^{N}}(f(z)-f(z-x)) d \mu^{t}(x) & \\
& =\int_{0}^{t}\left\{\int_{R^{N}}(f(z)-f(z-y))-\left(f^{\prime}(z) \cdot \tau(y)\right) d \nu^{s}(y)\right\} d s .
\end{aligned}
$$

Setting $z=0$ in (4.9) gives us (4.7) for $f \in C_{\infty}\left(R^{N}\right)$, after we note that $\nu^{s}$ is symmetric so $\int f^{\prime}(z) \cdot \tau(y) d \nu^{s}=0$.

Suppose now that $f$ is bounded, measurable and constant on $B(a)$ for some $a>0$. Let $\nu$ be the measure $\int_{0}^{t} \nu^{s} d s$. We note that

$$
\nu\left(B(a)^{c}\right)<\int_{0}^{t}\left(\mu^{s}\left(B(a / 2)^{c}\right)+M_{X}\left(B(a / 2)^{c}\right)\right) d s \leqslant t\left(1+M_{X}\left(B(a / 2)^{c}\right)\right) .
$$

Let $f_{n}$ be a sequence of bounded infinitely differentiable functions which are constant on $B(a)$ and such that $f_{n}(x)-f_{n}(0) \rightarrow f(x)-f(0)$ in $L^{1}\left(\nu+\mu^{t}\right)$. Since (4.7) is valid for $f_{n}$ we conclude it is valid for $f$. Q.E.D.

COROLLARY 4.1. Let $f$ be a bounded measurable real valued function which is constant in a neighbourhood of 0 . Let $F(s)=\int(f(0)-f(-x)) d \mu^{s}(x)$. Then $F$ is a continuous and differentiable function for $s \geq 0$ with

$$
F^{\prime}(s)=\int(f(0)-f(-x)) d \nu^{s}(x) \text {. }
$$

Proof. This follows immediately from (4.7).

THEOREM 4.1. Let $X$ be a symmetric infinitely divisible random vector in $R^{N}$. Suppose the Levy-Khinchine measure $M_{X}$ is in $U_{N}$; then $X$ is a symmetric unimodal random vector in $R^{N}$. 
Proof. Suppose first the special case that $M_{X}\left(R^{N}\right)$ is a finite constant and let $\left\|M_{X}\right\|$ denote this constant. Let $\mu_{X}$ stand for the distribution of $X$. It is well known that $\mu_{X}$ can be written as the mixture

$$
\mu_{X}=\exp ^{\left(-\left\|M_{X}\right\|\right)} \sum_{k=0}^{\infty} \frac{1}{k !}\left(M_{X}\right)^{k}
$$

where $\left(M_{X}\right)^{k}$ stands for the $k$-fold convolution of $M_{X}$ with itself. (See [9, p. 79].) For convenience rewrite (4.10) in symbolical form as

$$
\mu_{X}=\exp ^{\left(-\left\|M_{X}\right\|\right)} \exp ^{M_{X}} \text {. }
$$

Now each measure $\left(M_{X}\right)^{k}$ is in $U_{N}$ by Theorem 3.2 (which clearly extends to finite measures in $U_{N}$ ). Since $U_{N}$ is closed under mixtures we conclude $\mu_{X}$ is in $U_{N}$ in the special case $\left\|M_{X}\right\|<\infty$.

For the case with $\left\|M_{X}\right\|=\infty$ we consider the representation

$$
M_{X}=\int_{W_{N}} \lambda_{K} d \nu_{X}\left(\lambda_{K}\right)
$$

which follows from (2.1), where $\nu_{\mathrm{X}}$ is a measure on $W_{N}$. For any $r>0$ we know that $F(r) \equiv M_{X}\left(B(r)^{c}\right)<\infty$. We conclude from (4.12) that for any $a>0$ we have $\nu_{X}\left\{\lambda_{K} \mid \lambda_{K}\left(B(r)^{c}\right)>1 / a\right\} \leqslant a F(r)$. Let us now define a sequence of finite measures $\nu_{n}$ on $W_{N}$ by

$$
\nu_{n}(A)=\nu_{X}\left(A \cap\left\{\lambda_{K} \mid \lambda_{K}\left(B(1 / n)^{c}\right) \geqslant 1 / 2\right\}\right) .
$$

Let $M_{n}$ be the measures on $R^{N}$ defined by

$$
M_{n}=\int_{W_{N}} \lambda_{K} d \nu_{n}\left(\lambda_{K}\right)
$$

Clearly $M_{n}$ are finite measures in $U_{N}$ with $M_{n}\left(R^{N}\right) \leqslant 2 F(1 / n)$.

It is trivial to see that for all $a>0$ we have $M_{n} \rightarrow^{w} M$ on $B(a)^{c}$. Using [9, p. 80] we then see that $X_{n} \rightarrow{ }^{D} X$ where $X_{n}$ is the symmetric infinitely divisible random vector with Levy-Khinchine measure $M_{n}$. Now $X_{n}$ is symmetric unimodal by the special case first considered in the proof of this theorem. We conclude $X$ is symmetric unimodal by applying Theorem 3.1. Q.E.D.

COROLlaRy 4.2. Let $X$ be a symmetric infinitely divisible random vector in $R^{N}$ whose Levy-Khinchine measure $M_{X}$ factors as $\theta \times \Psi$, where $\theta$ is a measure on $(0, \infty)$ while $\Psi$ is a finite measure on $S_{N-1}$. Suppose further that $\theta$ has finite density $q(t)$ which is a decreasing function of $t$. Then $X$ is symmetric unimodal.

Proof. We claim that $M_{X}$ is in $U_{N}$. For any $s \in S_{N-1}$ let $\hat{s}$ stand for the set $\{s,-s\}$ and let $\hat{S}_{n-1}$ stand for the image of $S_{N}$ under the map $s \rightarrow \hat{s}$. Let $\hat{\Psi}$ stand for the image of the measure $\Psi$ under this map. Finally for any $\hat{s} \in \hat{S}_{N-1}$, let $p_{\hat{s}}$ stand for the measure on $R^{N}$ defined by 


$$
p_{\dot{s}}(B)=\theta\{t \mid t s \in B\}+\theta\{t \mid-t s \in B\}
$$

where $B$ is any Borel subset of $R^{N}$ and $\hat{s}=\{-s, s\}$. Clearly we can write $M_{X}$ as a mixture

$$
M_{X}=\int_{\hat{S}_{N-1}} p_{\hat{s}} d \hat{\Psi}(\hat{s})
$$

Now each measure $p_{\hat{s}}$ is symmetric and clearly has support on the line $L=\{t s \mid t \in R\}$. In other words, since $U_{N}$ is closed under mixing, we have reduced our claim to the one-dimensional case, where it is obvious. We conclude $M_{X}$ is indeed in $U_{N}$, and hence it follows from Theorem 4.1 that $X$ itself is symmetric unimodal. Q.E.D.

We are now going to apply our results to the study of multivariate stable densities.

Definition 4.2. A random vector $X$ in $R^{N}$ is said to be stable of index $\alpha$ if whenever $X_{1}, \ldots, X_{n}$ are independent random vectors distributed like $X$, then for some nonrandom vector $V_{n}$ we have that $n^{-1 / \alpha}\left(X_{1}+\cdots+X_{n}+\right.$ $\left.V_{n}\right)$ is distributed like $X$. It can be shown that $\alpha$ must be in $(0,2]$ and that $X$ is multivariate normal if $\alpha=2$. (See Feller [4] for some basic facts about stable random variables.)

It can be shown that every stable random vector is infinitely divisible. If $X$ is a symmetric stable random vector in $R^{N}$ of index $\alpha$ then its characteristic function $\phi_{X}(v)$ has the representation

$$
-\log \left(\phi_{X}(v)\right)=\int_{S_{N-1}}|(v \cdot s)|^{\alpha} d \Psi(s)
$$

where $\Psi$ is a finite measure on $S_{N-1}$ (see [12]). We can rewrite (4.16) as

$$
\log \left(\phi_{X}(v)\right)=k_{\alpha} \int_{S_{N-1}} \int_{0}^{\infty}(\cos (v \cdot t s)-1) \frac{1}{t^{1+\alpha}} d t d \Psi(s)
$$

for some positive constant $k_{\alpha}$, so we conclude that the Levy-Khinchine measure for $X$ factors as in Corollary 4.2, whence it follows that $X$ is symmetric and unimodal.

We now explore the notion of stochastic dominance in the context of symmetric infinitely divisible random vectors and derive results which are new even in one dimension (where they are easier to prove).

THEOREM 4.2. Let $\left(X_{i}(t) \mid t>0\right)$ be a time homogeneous stochastic process with symmetrically distributed independent increments with values in $R^{N}$ for $i=1,2$. (Assume also that $X_{i}(0)=0$ a.s.) Let $M_{i}$ be the Levy-Khinchine measure for $X_{1}(1)$ and suppose $M_{i} \in U_{N}$ for $i=1,2$. Then $M_{1} \succ M_{2}$ if and only if $X_{1}(t) \succ X_{2}(t)$ for all $t>0$. 
Proof. If $X_{1}(t) \succ X_{2}(t)$ for all $t>0$, then applying Corollary 4.1 to functions $f \in \mathscr{F}_{N}$ which are constant in a neighbourhood of 0 , we conclude that

$$
\int_{R^{N}}(f(0)-f(x)) d M_{1}(x)>\int_{R^{N}}(f(0)-f(x)) d M_{2}(x)
$$

for such $f$. To show that (4.18) holds for any $f \in \mathscr{F}_{N}$ choose $0<\theta<f(0)$ and define

$$
f_{\theta}(x)= \begin{cases}f(0)-\theta & \text { if } f(0)>f(x)>f(0)-\theta, \\ f(x) & \text { for all other } x .\end{cases}
$$

It is clear that $f_{\theta}$ is constant in a neighbourhood of 0 , so (4.18) is valid for $f_{\theta}$. Noting that $f_{\theta}(0)-f_{\theta}(x) \uparrow f(0)-f(x)$ as $\theta \rightarrow 0$, we conclude that (4.18) is valid for all $f \in \mathscr{F}_{N}$, so $M_{1}>M_{2}$.

We now argue the reverse implication. Suppose $M_{1}>M_{2}$ and let $\theta>0$. We shall show that $X_{1}((1+\theta) t) \succ X_{2}(t)$ for all $t>0$. (Note that $X_{1}(1+\theta)$ has Levy-Khinchine measure $(1+\theta) M_{i}$.) We denote by $\mu_{i}^{t}$ the distribution of $X_{i}(t)$ and by $\nu_{i}^{t}$ the convolution of $\mu_{i}^{t}$ with $M_{i}$ for $i=1,2$.

Suppose now that $f \in \mathscr{F}_{R^{N}}$. By [3] we have that

$$
f(x)<\exp \left(-c\|x\|_{2}\right) \text { on }\left\{x \mid\|x\|_{2}>d\right\}
$$

for some $c, d>0$. From (4.20) we conclude

$$
\int_{L} f(x) d \operatorname{Vol}_{L}(x)<\infty
$$

for any linear subspace $L$ of $R^{N}$.

Consider now the inequality

$$
\int_{R^{N}}(f(0)-f(x)) d \mu_{1}^{t(1+\theta)}(x)>\int_{R^{N}}(f(0)-f(x)) d \mu_{2}^{t}(x)
$$

which we wish to establish for all $f \in \mathscr{F}_{N}$ and all $t \geqslant 0$. Our procedure will be to establish (4.22) for successively larger classes of functions by a step-wise procedure. Let us make the following definitions: For $\delta>0, \mathscr{F}_{\delta}$ stands for $\left\{f \mid f \in \mathscr{F}_{N}, \int_{R^{N}}(f(0)-f(x)) d M_{1}(x)>\delta\right\}$. For $\delta, a>0, \mathscr{F}_{\delta, a}$ stands for the set $\left\{f \mid f \in \mathscr{F}_{\delta}, f \equiv 1\right.$ on $\left.B(a)\right\}$. For $\delta, a, k>0, \mathscr{F}_{\delta, a, k}$ stands for the set $\left\{f\left|f \in \mathscr{F}_{\delta, a},\right| f(x)-f(y) \mid<k\|x-y\|_{2}\right.$ for $\left.x, y \in R^{N}\right\}$. First we claim that if (4.22) holds for all elements of $\mathscr{F}_{\delta}$ for any $\delta>0$, then (4.22) holds for all elements of $\mathscr{F}_{N}$. This is obvious since for any nontrivial $M_{1}$, it is certainly true that $\int_{R^{N}}(f(0)-f(x)) d M_{1}(x)>\delta>0$ for any $f \in \mathscr{F}_{N}$ and $\delta$ sufficiently small. Next we claim that if (4.22) holds for all elements of $\mathscr{F}_{\delta, a}$ for any $\delta, a>0$ then (4.22) holds for all elements of $\mathscr{F}_{\delta^{\prime}}$, for any $\delta^{\prime}>0$. This follows since for $f \in \mathscr{F}_{\delta^{\prime}}, 0<\theta<1$, and $a$ sufficiently small, we have $B(a) \subset$ $\{x \mid f(x)>1-\theta\}$. Defining $f_{\theta}$ as in (4.19), we have $f_{\theta} \in \mathscr{F}_{\delta^{\prime} / 2, a}$ for $\theta$ 
sufficiently small ( $a$ depending on $\theta$ ). Now assuming (4.22) valid for $\mathscr{F}_{\delta^{\prime} / 2, a}$ we conclude (4.22) is valid for $f$ by letting $\theta \rightarrow 0$.

Our last claim is that if (4.22) holds for $\mathscr{F}_{\delta, a, k}$ for any $\delta, a, k>0$ then (4.22) holds for $\mathscr{F}_{\delta^{\prime}, a^{\prime}}$ for any $\delta^{\prime}, a^{\prime}>0$. We show this by first letting $h(x)$ be a fixed element of $\mathscr{F}_{N}$ such that $h(x) \equiv 1$ on $B(1 / 2)$ and $h(x) \equiv 0$ on $B(1)^{c}$. We may also assume that for some $k_{0}>0$ we have $|h(x)-h(y)| \leqslant k_{0}\|x-y\|_{2}$ for $x, y \in R^{N}$. We define $h_{n}(x)=(n)^{N} h(n x)$ and note that $\left|h_{n}(x)-h_{n}(y)\right|<$ $(n)^{N+1} k_{0}\|x-y\|$ for $x, y \in R^{N}$. Suppose now that $f \in \mathscr{F}_{\delta^{\prime}, a^{\prime}}$ for $\delta^{\prime}, a^{\prime}>0$. We define $f_{n}=f * h_{n}$ (which exists by 4.21). It is clear that $f_{n}(x) \rightarrow f(x)$ for all $x \in R^{N}$, and that $f_{n} \in \mathscr{F}_{N}$. (We know that $f$ is a logarithmically concave function by [3] or [10].) It is also clear that $f_{n}$ is identically 1 on $B\left(a^{\prime} / 2\right)$ for $n$ sufficiently large. Noting that $\sup _{n}\left|f_{n}(0)-f_{n}(x)\right| \leqslant f(0)$ for all $x$ we conclude that

$$
\int_{R^{N}}\left(f_{n}(0)-f_{n}(x)\right) d M_{1}(x) \rightarrow \int_{R^{N}}(f(0)-f(x)) d M_{1}(x)
$$

by the Lebesgue dominated convergence theorem. It follows that

$$
\int_{R^{N}}\left(f_{n}(0)-f_{n}(x)\right) d M_{1}(x)>\delta^{\prime} / 2
$$

for $n$ sufficiently large. Since $\left|f_{n}(x)-f_{n}(y)\right| \leqslant(n)^{N+1} k_{0}\|x-y\|_{2}$ for $x, y \in$ $R^{N}$, we have that $f_{n}$ is in $\mathscr{F}_{\delta^{\prime} / 2, a^{\prime} / 2,(n)^{N+1} k_{0}}$. So if we assume (4.22) valid for all $\mathscr{F}_{a, \delta, k}$ we conclude (4.22) is valid for $f$ by letting $n \rightarrow \infty$.

At this point we have convinced ourselves that it is sufficient to demonstrate (4.22) for $f \in \mathscr{F}_{\delta, a, k}$ for $\delta, a, k>0$. Suppose by way of contradiction that (4.22) is false for some $t>0$ and some $f \in \mathscr{F}_{\delta, a, k}$. Then the set $V=\{t \mid t$ $>0$ and (4. 22) is false for some $f$ in $\left.\mathscr{F}_{\delta, a, k}\right\}$ is nonempty. Let $T=\inf (V)$. If $T=0$ then there exists a sequence $t_{N} \downarrow 0$ and $f_{n} \in \mathscr{F}_{\delta, a, k}$ such that (4.22) fails for $f_{n}$ at $t_{n}$.

Now by $[9$, p. 51] we know that for $i=1,2$,

$$
\begin{aligned}
\lim _{t \rightarrow 0} \sup _{f \in \mathscr{F}_{\delta, a, k}} & \mid \frac{1}{t} \int_{R^{N}}(f(0)-f(x)) d \mu_{i}^{t}(x) \\
& -\int_{R^{N}}(f(0)-f(x)) d M_{i}(x) \mid=0 .
\end{aligned}
$$

We conclude from (4.23) and the fact that (4.22) is false for $t_{n}$ and $f_{n}$ that

$$
\begin{aligned}
0<\delta< & <\limsup _{n \rightarrow \infty} \int_{R^{N}}\left(f_{n}(0)-f_{n}(x)\right)(1+\theta) d M_{1}(x) \\
& <\liminf _{n \rightarrow \infty} \int_{R^{N}}\left(f_{n}(0)-f_{n}(x)\right) d M_{2}(x) .
\end{aligned}
$$

Clearly (4.24) contradicts the assumption that $M_{1}>M_{2}$; we conclude that $T>0$. 
It is easy to show that $\mu_{1}^{s(1+\theta)}>\mu_{2}^{s}$ for all $s \in(0, T)$ if $T$ is finite by arguing as above.

We will show that $T=\infty$. Suppose not, then for some $f \in \mathscr{F}_{\delta, a, k}$ we have (4.22) valid for $t \in[0, T)$ but fails when $t=T$. Let

$$
F_{1}(s)=\int_{R^{N}}(f(0)-f(-x)) d \mu_{1}^{s(1+\theta)}(x)
$$

and let

$$
F_{2}(s)=\int_{R^{N}}(f(0)-f(-x)) d \mu_{2}^{s}(x) .
$$

We know that $F_{i}(s)$ are continuous and differentiable functions by Corollary 4.1. We have already shown that $F_{1}^{\prime}(0)>F_{2}^{\prime}(0)$. It follows that $F_{1}(s)>F_{2}(s)$ for $s \in\left[0, t_{1}\right)$ for some $t_{1}>0$. Using Corollary 4.1 again, and noting that $\mu_{1}^{s(1+\theta)}>\mu_{2}^{s}$ for $s \in(0, T)$ we see that $F_{1}(T)>F_{2}(T)$ which is a contradiction. Q.E.D.

5. Other notions of unimodality and some counterexamples. In this section we consider other possible definitions of unimodality for symmetric random vectors in $R^{N}$. We end with a counterexample relating to symmetric stable random vectors.

The earliest relevant definition is due to Anderson [1] who defined a random variable $X$ in $R^{N}$ to be symmetric unimodal if it had a density function $f$ with respect to $\operatorname{Vol}_{N}$ such that $f$ was symmetric and the sets $\{x \mid f(x) \geqslant \mu\}$ were convex for all $\mu \in[0, \infty)$. This notion of unimodality is not closed under convolution, as he himself was able to show.

In [13] Sherman introduced a more general definition. According to him a random variable $X$ in $R^{N}$ was symmetric unimodal if $X$ had a density function $f$ with respect to $\operatorname{Vol}_{N}$ such that $\lim _{n \rightarrow \infty}\left\|f-f_{n}\right\|_{1}=0$, where $f_{n}$ was some finite mixture $\Sigma_{1}^{k_{N}} \alpha_{i} \lambda_{K_{i}}$ with $K_{i}$ compact, symmetric, convex sets in $R^{N}$ containing 0 in their interior, while $\|h\|=\max \left\{\|h\|_{\infty},\|h\|_{1}\right\}$. (\| $\|_{\infty}$ is the supremum norm and \|\|$_{1}$ is the $L^{1}\left(\mathrm{Vol}_{N}\right)$ norm. $)$ This notion of unimodality is closed under convolution, but it is easy to see that it is not closed under weak convergence.

A more recent definition is due to Olshen and Savage [8]. They define a random vector $X$ in $R^{N}$ to be $\alpha$-unimodal about 0 if for all real valued bounded, nonnegative, Borel functions $g$ on $R^{N}$ the function $t^{\alpha} E(g(t x))$ is decreasing as $t$ increases, $t \in[0, \infty)$. If $X$ has a density function with respect to $\mathrm{Vol}_{L}$ for some linear subspace $L$ of $R^{N}$ of dimension $k$, then according to [6] $X$ is $\alpha$-unimodal about 0 if and only if there exists some version $f$ of the density such that the function $(s x)^{k-\alpha} f(s x)$ is decreasing for any $x \in L$ as $s$ increases, $s \in[0, \infty)$. The notion of $\alpha$-unimodality is closed under weak convergence, but not under convolution (the convolution of $\alpha$-unimodal 
probability measures with $\beta$-unimodal probability measures is only $\alpha+\beta$ unimodal).

Comparing the above notions of unimodality to our own we see clearly that any symmetric random vector $X$ which is unimodal according to Anderson or Sherman is unimodal in our sense as well. On the other hand if a symmetric random vector $X$ in $R^{N}$ has distribution $\mu$ which is a mixture of measures all in $U_{L}$ where $L$ is a (variable) linear subspace of $R^{N}$ with $\operatorname{dim}(L)<k$, then we easily conclude that $X$ is $k$-unimodal.

For symmetric random vectors $X$ in $R^{N}$ with density with respect to $\mathrm{Vol}_{N}$ the condition that $X$ be $N$-unimodal (i.e. that some version of the density of $X$ is decreasing along rays from the origin) seems to be the most general notion of unimodality possible. It is clear that any symmetric random vector in $R^{N}$ which is unimodal in our sense is $N$-unimodal. We now give an example showing the converse is not true.

EXAMPLe 5.1. Let $K=\operatorname{conv}(0,0),(1,1),(1,-1)$, let $A=K \cup-K$, and let $f=I_{A} / \operatorname{Vol}_{2}(A)$. It is clear that $f$ is 2-unimodal. To show that $f d \mathrm{Vol}_{2}$ is not in $U_{2}$ we consider the function

$$
F(s)=\int_{-1 / 2}^{+1 / 2} \int_{-1 / 2}^{+1 / 2} f(x+s, y) d x d y .
$$

We evaluate $F(0)=\frac{1}{4}$ and $F\left(\frac{1}{2}\right)=\frac{1}{2}$. However, if $f$ is in $U_{2}$ then it is clear from Corollary 2.1 that $F(s)$ is decreasing as $s$ increases, $s \in[0, \infty)$.

In [5] Ghosh introduces another definition of multivariate unimodality (called type 1 ) by defining a random vector $X$ in $R^{N}$ to be unimodal with vertex at 0 if for every $a \in R^{N}$ we have $a \cdot X$ has a one-dimensional unimodal distribution with vertex at 0 (i.e. concave to the left of 0 and convex to the right of 0 ). He claimed his definition was motivated by geometrical considerations, however, as the following example shows, densities which are unimodal in the Ghosh sense need not even be $N$-unimodal.

EXAMPLE 5.2. For any $\varepsilon \in(0,1)$ consider the density $f(x, y)$ in $R^{2}$ defined by $f(x, y)=K_{\varepsilon}\left(I_{B(1)}+\varepsilon\left(x^{2}+y^{2}\right)\right)$, where $K_{\varepsilon}$ is a normalizing constant. A trivial calculation shows that all one-dimensional marginals of $f$ are symmetric unimodal, hence $f$ is unimodal in the Ghosh sense.

We conclude with an instructive example of a stable density.

EXAMPLE 5.3. Consider the function

$$
f(x, y)=\frac{1}{\pi^{2}}\left(\frac{1}{1+x^{2}}\right)\left(\frac{1}{1+y^{2}}\right) .
$$

It is the density of a bivariate Cauchy random vector, both coordinates independent and identically distributed, and gives an example of a random vector which is symmetric unimodal in our sense, but is not unimodal according to Anderson's definition. (Note that the curves $\left(1+x^{2}\right)\left(1+y^{2}\right)=$ 
$C$ are starlike in shape, but not convex, if $C$ is large.)

ADDED IN PROOF. R. Ahmad and A. M. Abonammoh have announced the solution of a conjecture of Sherman which provides another characterization of the class of symmetric unimodal random vectors. (See I. M. S. Bulletin 6 (1977), 38.) Dharmadhikari and Jogdeo [Ann. Math. Statist. 4 (1976), 607-613] have done related work.

\section{REFERENCES}

1. T. W. Anderson (1955), The integral of a symmetric unimodal function over a symmetric convex set and some probability inequalities, Proc. Amer. Math. Soc. 6, 170-176. MR 16, 1005.

2. C. Borell (1975), Convex set functions in d-space, Period. Math. Hungar. 6, 111-136.

3. J. C. Davidovic, B. I. Korenbljum and B. I. Hacet (1969), A certain property of logarithmically concave functions, Dokl. Akad. Nauk SSSR 185, 1215-1218 = Soviet Math. Dokl. 10, 477-480. MR 39 \#2924.

4. W. Feller (1966), An introduction to probability theory and its applications. Vol. II, Wiley, New York. MR 35 \# 1048.

5. P. Ghosh (1974), On generalized unimodality, Comm. Statist. 3, 567-580. MR 50 \# 1374.

6. E. Hille and R. S. Phillips (1957), Functional analysis and semi-groups, Amer. Math. Soc. Colloq. Publ., vol. 31, rev. ed., Amer. Math. Soc., Providence, R. I. MR 19, 664.

7. I. A. Ibragimov and Y. V. Linnik (1971), Independent and stationary sequences of random variables, "Nauka", Moscow, 1965; English transl., Noordhoff, Groningen. MR 34 \#2049; 48 \#1287.

8. A. R. Olshen and L. J. Savage (1970), A generalized unimodality, J. Appl. Probabilities 7, 21-34. MR 41 \#6274.

9. K. R. Parthasarathy (1967), Probability measures on metric spaces, Probability and Mathematical Statistics, No. 3, Academic Press, New York and London. MR 37 \#2271.

10. A. Prékopa (1973), On logarithmically concave measures and functions, Acta. Math. Acad. Hungar. 335-343.

11. A. W. Roberts and D. E. Varberg (1973), Convex functions, Academic Press, New York.

12. M. Schilder (1970), Some structure theorems for the symmetric stable laws, Ann. Math. Statist. 41, 412-421. MR 40 \# 8122.

13. S. Sherman (1955), A theorem on convex sets with applications, Ann. Math. Statist. 26, 763-767. MR 17, 655.

14. A. C. Zaanen (1958), An introduction to the theory of integration, North-Holland, Amsterdam. MR 20 \#3950.

Department of Statistics, Univeristy of New South Wales, Kensington, N.S.W. 2033, Australia

Current address: Department of Mathematics, Sir George Williams Campus, Concordia University, Montreal, Quebec, Canada 